\title{
Probing the Wave Function of Shallow Li and Na Donors in ZnO Nanoparticles
}

\author{
Serguei B. Orlinskii and Jan Schmidt \\ Huygens Laboratory, Leiden University, P.O. Box 9504, 2300 RA Leiden, The Netherlands \\ Pavel G. Baranov \\ A.F. Ioffe Physico-Technical Institute, RAS, 194021 St. Petersburg, Russia \\ Detlev M. Hofmann \\ I. Physikalisches Institut, Heinrich-Buff Ring 16, Justus-Liebig Universität Giessen, D-35392 Giessen, Germany \\ Celso de Mello Donegá and Andries Meijerink \\ Debye Instiiute, Utrecht University, Utrecht, The Netherlands
}

(Received 27 September 2003; published 28 January 2004)

\begin{abstract}
Electron paramagnetic resonance and electron nuclear double resonance (ENDOR) experiments on $\mathrm{ZnO}$ nanoparticles reveal the presence of shallow donors related to interstitial $\mathrm{Li}$ and $\mathrm{Na}$ atoms. The shallow character of the wave function is evidenced by the multitude of ${ }^{67} \mathrm{Zn}$ ENDOR lines and further by the hyperfine interactions with the ${ }^{7} \mathrm{Li}$ and ${ }^{23} \mathrm{Na}$ nuclei that are much smaller than for atomic lithium and sodium. In the case of the Li-doped nanoparticles, an increase of the hyperfine interaction with the ${ }^{7} \mathrm{Li}$ nucleus and with the ${ }^{1} \mathrm{H}$ nuclei in the $\mathrm{Zn}(\mathrm{OH})_{2}$ capping layer is observed when reducing the size of the nanoparticles. This effect is caused by the confinement of the shallow-donor $1 s$-type wave function that has a Bohr radius of about $1.5 \mathrm{~nm}$, i.e., comparable to the dimension of the nanoparticles.
\end{abstract}

DOI: 10.1103/PhysRevLett.92.047603

$\mathrm{ZnO}$, with a direct band gap of 3.3-3.4 eV, attracts considerable attention because of its promising applications for UV light-emitting diodes and diode lasers. A problem with $\mathrm{ZnO}$ is that it can easily be made $n$ type [1], but that it is difficult to dope $\mathrm{ZnO} p$ type although recent reports suggest that $p$ doping is possible with $\mathrm{N}$ or As $[2-4]$. The $n$-type doping has traditionally been attributed to native defects [5]. However, a recent first-principles study revealed that none of the native defects exhibit characteristics consistent with a high concentration of shallow donors [6], and it was concluded that the observed $n$-type conductivity can be caused only by impurities that are unintentionally incorporated. Quite unexpectedly, it was proposed by Van de Walle [7] that interstitial H behaves as a shallow donor. This theoretical prediction was confirmed recently by the observation of the electron nuclear double resonance (ENDOR) signal of $\mathrm{H}$ in the electron paramagnetic resonance (EPR) signal of this shallow donor [8].

The observation that interstitial $\mathrm{H}$ acts as a shallow donor suggests that similar effects may be expected upon the introduction of other group-I elements. Indeed, in a recent paper Park et al. [9], on the basis of theoretical calculations, predicted that $\mathrm{Li}$ and $\mathrm{Na}$ prefer interstitial sites over substitutional sites in $\mathrm{ZnO}$ and behave as shallow donors. To check this idea we have started an investigation by high-frequency EPR and ENDOR spectroscopy of $\mathrm{ZnO}$ nanoparticles doped with $\mathrm{Li}$ and $\mathrm{Na}$. The reason to study nanometer-sized particles of $\mathrm{ZnO}$ is twofold. First, it is relatively easy to prepare this material and to dope it with $\mathrm{Li}$ and $\mathrm{Na}$. Second, it allows us not only to
PACS numbers: 76.30.Da, 61.72.Vv, 71.55.Gs, 76.70.Dx

identify the shallow donor via the ENDOR signals of the nuclear spin of the binding core and the nuclear spins of the surrounding ${ }^{67} \mathrm{Zn}$ ions, but also to probe the effects of confinement on its spatially extended wave function by varying the particle size in the quantum-size regime.

The preparation of the freestanding hydroxyl-capped $\mathrm{ZnO}$ nanocrystals in the form of dry powders was achieved using a modified version of methods reported in the literature [10-12]. Our method was based on the hydrolysis of $\mathrm{Zn}^{2+}$ ions in absolute alcohols (ethanol or 1-butanol), using either $\mathrm{LiOH} \cdot \mathrm{H}_{2} \mathrm{O}$ for the $\mathrm{Li}$-doped nanocrystals or $\mathrm{NaOH}$ for the Na-doped nanocrystals. The size of the nanocrystals ranged from 3.2 to $4.5 \mathrm{~nm}$ and was controlled by the growth duration $(5 \mathrm{~min}$ to 1 day). The average diameter of the nanocrystals was estimated by $\mathrm{x}$-ray powder diffraction, based on the peak broadening due to the finite crystallite sizes (Scherrer's equation), and by UV-visible absorption spectroscopy, based on the size dependence of the band gap owing to quantum-size effects and using a calibration curve [10].

The EPR and ENDOR experiments were performed at $1.6 \mathrm{~K}$ using a pulsed $95 \mathrm{GHz}$ EPR spectrometer [13]. The great advantage of working at this high microwave frequency is that a high spectral resolution is obtained in the EPR as well as in the ENDOR spectra. In addition, the use of pulsed microwave techniques facilitates considerably the observation of the ENDOR spectra [13]. The EPR spectra were recorded by monitoring the electron spin echo (ESE) signal following a microwave $\pi / 2$ - and a $\pi$-pulse sequence. The ENDOR spectra were obtained 
by monitoring the intensity of the stimulated echo, following three microwave $\pi / 2$ pulses, as a function of the frequency of a radiofrequency pulse applied between the second and third microwave pulses [14].

Figure 1 shows the ESE-detected EPR spectrum of a dry powder sample of Li-doped $\mathrm{ZnO}$ nanoparticles with an average diameter of $3.4 \mathrm{~nm}$ under continuous ultraviolet irradiation at $1.6 \mathrm{~K}$. The signal labeled I at $3.4600 \mathrm{~T}$ with a linewidth of $6.0 \mathrm{mT}$ is assigned to the interstitial $\mathrm{Li}$ donor. Its average $g$ value $g_{\text {av }}=1.9666$ differs somewhat from the $g_{\|}=1.9569$ and $g_{\perp}=1.9552$ values obtained for the interstitial-H donor in a single crystal of $\mathrm{ZnO}$ [8]. The linewidth, however, corresponds very well with $g_{\|}-$ $g_{\perp}=0.0017$ obtained for the interstitial-H donor and taking into account the random character of the powder sample. The EPR signal labeled II at $3.3575 \mathrm{~T}$ is assigned to a Na-related center, whereas the signal labeled III is identified as originating from the deep $\mathrm{Li}_{\mathrm{Zn}}$ acceptor $[15,16]$. The arguments that lead to the assignments of signals II and III will be presented later in this Letter. We will first concentrate on the EPR signal I assigned to the shallow Li donor.

The EPR signal $I$ in Fig. 1 is assigned to a donor because $g_{\text {av }}$ is smaller than the $g$ value of a free electron. The shallow character becomes clear from the dependence of $g_{\text {av }}$ on the size of the nanoparticles. We find that $g_{\text {av }}=1.9628$ for $4.4 \mathrm{~nm}$ particles increases to $g_{\text {av }}=$ 1.9670 for $3.2 \mathrm{~nm}$ particles. This shift towards the freeelectron $g_{e}$ value has been observed by Zhou et al. [17] and is caused by the confinement of the H-like $1 s$-type wave function of shallow donors when the Bohr radius becomes comparable to the size of the nanoparticles. The effect is explained by the reduction of the admixture of valence-band states and higher-lying conduction bands by the increase of the band gap energy and the energy of

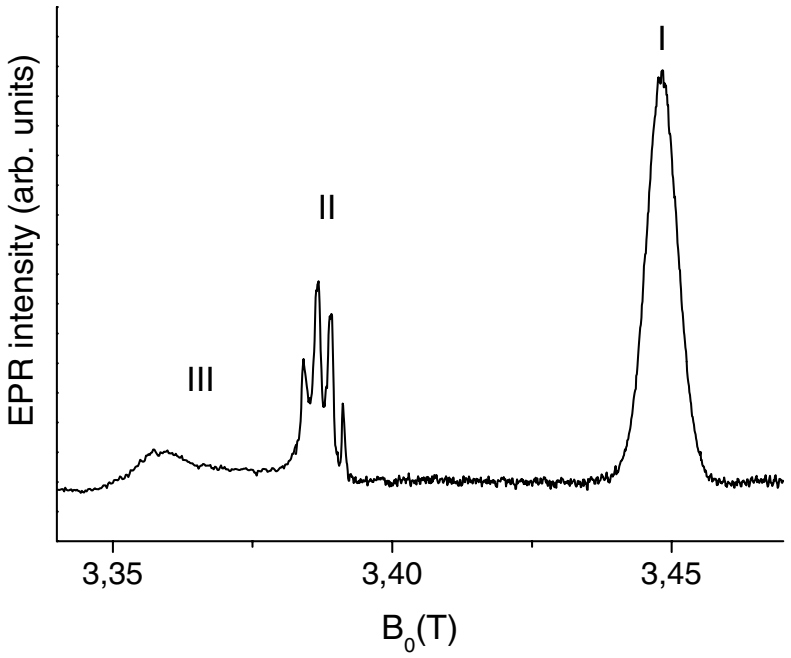

FIG. 1. The ESE-detected EPR spectrum at $94.9 \mathrm{GHz}$ of a dry powder sample of Li-doped $\mathrm{ZnO}$ nanoparticles with an average diameter of $3.4 \mathrm{~nm}$ under continuous UV irradiation. higher-lying conduction bands upon the reduction of the size of the nanoparticles [18].

In Fig. 2, the ENDOR signals are presented as obtained on the EPR signal I of the shallow donor. To understand these results, we consider the isotropic hyperfine (hf) interaction or Fermi contact term $a_{i}=$ $(8 \pi / 3) g_{e} \beta_{e} g_{n i} \beta_{n}\left|\Psi\left(r_{i}\right)\right|^{2}$, which reflects the spin density of the donor electron wave function $(\Psi)$ at the site of the nucleus $\left(r_{i}\right)$. Here $g_{e}$ is the electronic $g$ factor, $\beta_{e}$ is the electronic Bohr magneton, $g_{n i}$ is the $g$ factor of nucleus $i$, and $\beta_{n}$ is the nuclear magneton. The related ENDOR transition frequencies are $\nu_{\mathrm{ENDOR} i}=$ $h^{-1}\left|g_{n i} \beta_{n} B_{0} \pm a_{i} / 2\right|$, where each nucleus $i$ gives rise to two ENDOR transitions symmetrically placed around its nuclear Zeeman frequency $g_{n i} \beta_{n} B_{0} / h$ when the quadrupole interaction is neglected and when $a_{i}<g_{n i} \beta_{n} B_{0}$.

First, it is seen in Fig. 2 that symmetrically around the Zeeman frequency of ${ }^{67} \mathrm{Zn}(I=5 / 2$, abundance $4.1 \%)$ at $9.2 \mathrm{MHz}$ a broad, an unresolved set of ENDOR lines of ${ }^{67} \mathrm{Zn}$ spins is present. From the multitude of lines, it is clear that we are indeed dealing with the electron of a shallow donor that interacts with a large number (about 20) of ${ }^{67} \mathrm{Zn}$ nuclei. Second, it is seen that symmetrically around the Zeeman frequency of ${ }^{7} \mathrm{Li}(I=3 / 2$, abundance $92.5 \%$ ) at $57.1 \mathrm{MHz}$, two ENDOR lines are present, separated by $90 \mathrm{kHz}$, that are assigned to ${ }^{7} \mathrm{Li}$. These signals are taken as proof that $\mathrm{Li}^{+}$forms an interstitial core for the shallow-donor electron in the $\mathrm{ZnO}$ nanoparticle.

The observation of the ${ }^{7} \mathrm{Li}$ ENDOR signals allows us to measure directly the effect of confinement on the wave function of the shallow Li donor. One would expect that the density of the wave function at the $\mathrm{Li}^{+}$core will vary according to $R^{-3}$ ( $R$ is the size of the core of the nanoparticle) assuming that only a very small fraction of the wave function density penetrates into the $\mathrm{Zn}(\mathrm{OH})_{2}$

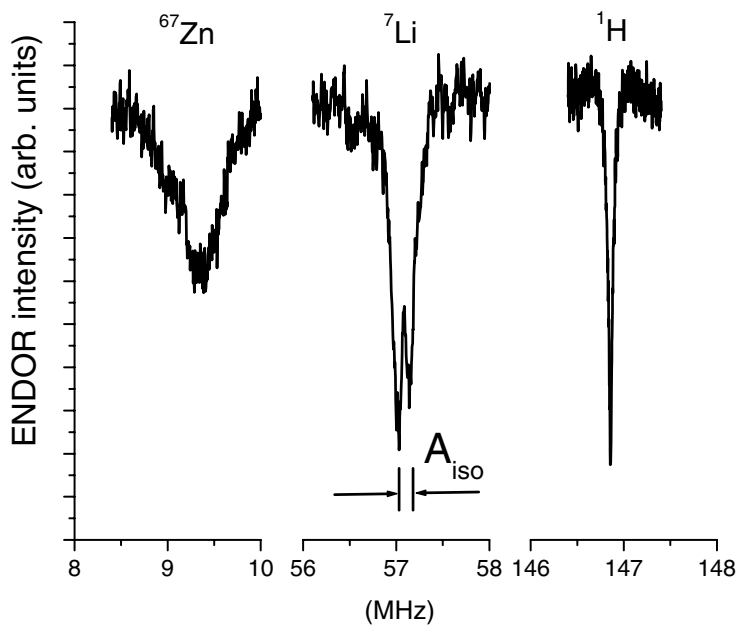

FIG. 2. The ENDOR transitions of the ${ }^{67} \mathrm{Zn},{ }^{7} \mathrm{Li}$, and ${ }^{1} \mathrm{H}$ nuclear spins observed in the EPR signal $I$ (see Fig. 1) of the shallow Li-related donor. 
capping layer and thus that the integrated density in the core remains almost constant when decreasing the volume of the nanoparticle. This is indeed what is observed. In Fig. 3, the observed ${ }^{7} \mathrm{Li}$ hf splitting is presented as a function of the size of the nanoparticle. In the same figure, a curve is plotted representing a $R^{-3}$ dependence which is given the observed value of $90 \mathrm{kHz}$ for a particle with a size of $4.4 \mathrm{~nm}$. It is seen that this curve predicts quite well the observed hf splitting of $220 \mathrm{kHz}$ for a particle with a size of $3.2 \mathrm{~nm}$.

To check whether interstitial $\mathrm{H}$ might be present as the core of a shallow donor in the $\mathrm{ZnO}$ nanoparticles, we have also carried out a search for ENDOR lines around the Zeeman frequency of ${ }^{1} \mathrm{H}$ at $147.5 \mathrm{MHz}$. It is seen in Fig. 2 that an ENDOR line is present with a width $\Delta \nu=$ $60 \mathrm{kHz}$ exactly at the Zeeman frequency of ${ }^{1} \mathrm{H}$. From the width we deduce a ${ }^{1} \mathrm{H}$ hf interaction smaller than $60 \mathrm{kHz}$. This should be compared to our previous observation on the $\mathrm{H}$-related shallow donor in a bulk crystal of $\mathrm{ZnO}$ where two ENDOR lines were found with a hf splitting of $1.4 \mathrm{MHz}$ [8]. We conclude that the observed ENDOR lines originate in the $\mathrm{H}$ atoms present in the $\mathrm{Zn}(\mathrm{OH})_{2}$ capping layer where the density of the electronic wave function is very small.

In Fig. 3, we also show the observed width of the ${ }^{1} \mathrm{H}$ ENDOR line when varying the particle size from 4.4 to $3.2 \mathrm{~nm}$. It is seen that the results can again be described by a $R^{-3}$ dependence. Here the theoretical $R^{-3}$ curve has been given the value of $30 \mathrm{kHz}$ for particles with a size of $4.4 \mathrm{~nm}$. Following an identical reasoning as for the size dependence of the ${ }^{7} \mathrm{Li} \mathrm{hf}$ splitting, one predicts that the

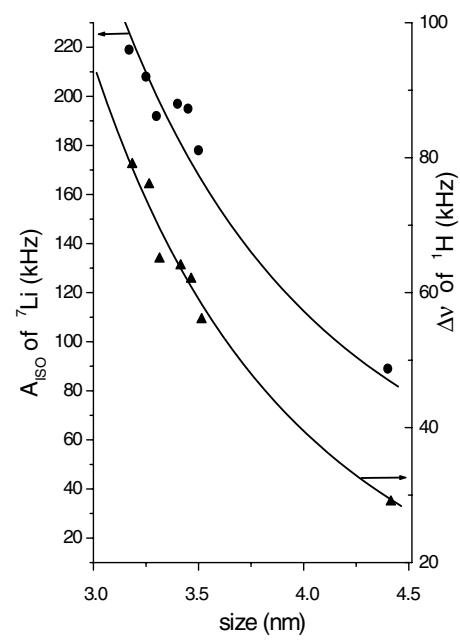

FIG. 3. The ${ }^{7} \mathrm{Li}$ hf splitting as observed in the ENDOR experiments (full black dots) and the linewidth of the ${ }^{1} \mathrm{H}$ ENDOR transition (full black triangles) both as a function of the size $R$ of the $\mathrm{ZnO}$ nanoparticles. The experimental results are compared to curves representing a $R^{-3}$ dependence. The $R^{-3}$ curve for ${ }^{7} \mathrm{Li}$ is given the value of $90 \mathrm{kHz}$ and the $R^{-3}$ curve for ${ }^{1} \mathrm{H}$ the value of $30 \mathrm{kHz}$ for particles with an average size of $4.4 \mathrm{~nm}$. density of the electronic wave function at the interface of the $\mathrm{ZnO}$ core and the $\mathrm{Zn}(\mathrm{OH})_{2}$ layer should also exhibit a $R^{-3}$ dependence. As a result, the distribution of the density of the wave function in the capping layer, as reflected in the width of the ${ }^{1} \mathrm{H}$ ENDOR line, should follow this dependence.

The question arises whether the introduction of $\mathrm{Li}$ in the $\mathrm{ZnO}$ nanoparticles also leads to the presence of a $\mathrm{Li}_{\mathrm{Zn}}$ deep acceptor as observed in Li-doped bulk $\mathrm{ZnO}$ crystals. It has been shown by an EPR and ENDOR study that $\mathrm{Li}_{\mathrm{Zn}}$ and $\mathrm{Na}_{\mathrm{Zn}}$ are deep acceptors with the hole located on adjacent oxygen atoms $[15,16]$. We have compared the line shape of the EPR signal III in Fig. 1 with a simulated curve using the known anisotropy of the $g$ tensor of the deep $\mathrm{Li}_{\mathrm{Zn}}$ acceptor $[15,16]$ and assuming that the $\mathrm{ZnO}$ nanoparticles are randomly oriented. This simulated curve corresponds very well with the observed line shape. We take this similarity as support for the contention that the EPR line III in Fig. 1 originates in the deep $\mathrm{Li}_{\mathrm{Zn}}$ acceptor.

The shape of the EPR signal labeled II in Fig. 1 with a $\mathrm{hf}$ interaction that is nearly isotropic suggests a hf interaction with a nucleus with spin $I=3 / 2$ with an almost $100 \%$ abundance. This observation favors a Na-related center and indeed the ENDOR study of this signal reveals two transitions at 4.2 and $72.0 \mathrm{MHz}$. The difference of these two frequencies is equal to the splitting of $2.4 \mathrm{mT}$ of the hf components in the EPR signal and their sum is equal to 2 times the Zeeman frequency of the ${ }^{23} \mathrm{Na}(I=$ $3 / 2$ ) nuclear spin. We assign the EPR signal to a $\mathrm{Na}_{Z \mathrm{nn}}-\mathrm{V}_{\mathrm{O}}$ deep center. The observed hf splitting of $A\left({ }^{23} \mathrm{Na}\right)=$ $2.4 \mathrm{mT}$ is about $7 \%$ of the hf constant for free $\mathrm{Na}^{0}$ [19]. We thus believe that the center is deep because for shallow

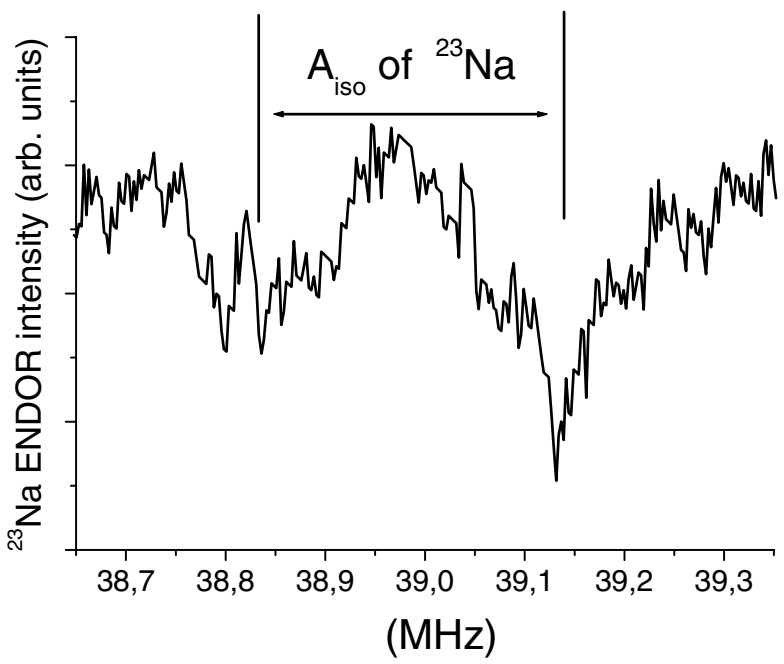

FIG. 4. The ENDOR transitions of ${ }^{23} \mathrm{Na}$ as observed in the EPR signal of the shallow donor in Na-doped $\mathrm{ZnO}$ nanoparticles with an average size of $3.0 \mathrm{~nm}$. The two ENDOR transitions are symmetrically placed around the Zeeman frequency of ${ }^{23} \mathrm{Na}$ at $38.97 \mathrm{MHz}$ with a splitting of $300 \mathrm{kHz}$. 
centers the hf structure constant is usually much less than $1 \%$ of the free ion value. The defect center is believed to be a deep donor. We note that the $\mathrm{Na}$ impurity may originate from the glass ware or from the compounds used for the preparation of the Li-doped $\mathrm{ZnO}$ nanoparticles.

To check whether interstitial $\mathrm{Na}$ can also act as a shallow donor in $\mathrm{ZnO}$, we have performed similar EPR and ENDOR experiments on $\mathrm{ZnO}$ nanoparticles that were prepared using $\mathrm{NaOH}$. In such $\mathrm{ZnO}$ nanoparticles with a diameter of $3.0 \mathrm{~nm}$ and under permanent $\mathrm{UV}$ illumination, we observe again three EPR signals in analogy to the Li-doped sample. First, an EPR signal similar to I in Fig. 1 with a $g_{\text {av }}=1.9592$ that is assigned to a shallow donor; second, a signal similar to II that is assigned to a deep $\mathrm{Na}_{\mathrm{Zn}}-\mathrm{V}_{\mathrm{O}}$ center; and, third, a signal similar to III that is assigned to the deep $\mathrm{Na}_{\mathrm{Zn}}$ acceptor $[15,16]$. In Fig. 4, the result is presented of an ENDOR experiment on the signal with $g_{\text {av }}=1.9592$, assigned to a shallow donor, which reveals two transitions with a splitting of $300 \mathrm{kHz}$ symmetrically placed around the Zeeman frequency of ${ }^{23} \mathrm{Na}$ at $38.9 \mathrm{MHz}$. We consider this as proof of the presence of a shallow donor related to interstitial $\mathrm{Na}$ in the $\mathrm{ZnO}$ lattice.

In summary, we have observed the presence of shallow donors in $\mathrm{ZnO}$ nanoparticles that are related to interstitial $\mathrm{Li}$ and $\mathrm{Na}$ atoms. We thus confirm the results of theoretical predictions that $\mathrm{Li}$ and $\mathrm{Na}$ can enter the $\mathrm{ZnO}$ lattice interstitially and can act as shallow donors [9]. The experimental evidence that interstitial $\mathrm{Li}^{+}$and $\mathrm{Na}^{+}$act as the binding core is the observation of the ENDOR signals of the $I=3 / 2$ nucleus of ${ }^{7} \mathrm{Li}$ and of the $I=3 / 2$ nucleus of ${ }^{23} \mathrm{Na}$ in the EPR signals. The shallow character of these donors is evidenced by the observed hf splittings of the ${ }^{7} \mathrm{Li}$ and ${ }^{23} \mathrm{Na}$ nuclei that are much smaller than the hf interactions of 364.9 and $927.1 \mathrm{MHz}$ for atomic lithium and sodium, respectively [19]. The observation in the case of the Li-doped nanoparticles of the multitude of ${ }^{67} \mathrm{Zn}$ ENDOR transitions, of the variation with size of the electronic $g$ value, of the ${ }^{7} \mathrm{Li}$ hf splitting, and of the width of the ENDOR transition of the ${ }^{1} \mathrm{H}$ nuclear spin in the $\mathrm{Zn}(\mathrm{OH})_{2}$ capping layer further shows that we are dealing with confinement effects on the $1 s$-type electronic wave function that has a Bohr radius in bulk $\mathrm{ZnO}$ comparable to the dimensions of the nanoparticles. In addition to the shallow interstitial $\mathrm{Li}$ and $\mathrm{Na}$ donor, we also observe the EPR signals of the deep, substitutional $\mathrm{Li}_{\mathrm{Zn}}$ and $\mathrm{Na}_{\mathrm{Zn}}$ acceptor. The EPR signal of a Na-related deep center is assigned to a substitutional $\mathrm{Na}_{\mathrm{Zn}}$ in combination with a neighboring $\mathrm{O}$ vacancy.

This work forms part of the research program of the Netherlands Foundation for Fundamental Research of Matter (F.O.M.) and the Technology Foundation STW, both with financial support from the Nederlandse Organisatie voor Wetenschappelijk Onderzoek (N.W.O.). Financial support from the SENTINEL Network in the framework of the 5th EC Science Program is acknowledged. P.G. B. acknowledges support by RFBR under Grant No. 03-02-17645 and the Project of RAS "Spindependent effects in solids and spintronics."

[1] S. B. Zhang, S.-H. Wei, and A. Zunger, J. Appl. Phys. 83, 3192 (1998), and references therein.

[2] M. Joseph, H Tabata, and T. Kawai, Jpn. J. Appl. Phys. 38, L1205 (1999).

[3] Y. R. Ryu et al., J. Cryst. Growth 216, 330 (2000).

[4] Y. Yan, S. B. Zhang, and S. T. Pantelides, Phys. Rev. Lett. 86, 5723 (2001).

[5] D. C. Look, J. M. Hemsky, and J. R. Sizelove, Phys. Rev. Lett. 82, 2552 (1999).

[6] A. F. Kohan, G.Ceder, D. Morgan, and Chris G. Van de Walle, Phys. Rev. B 61, 15019 (2000).

[7] C. G. Van de Walle, Phys. Rev. Lett. 85, 1012 (2000).

[8] D. M. Hofmann, A. Hofstaetter, F. Leiter, H. Zhou, F. Henecker, B. K. Meyer, S. B. Orlinskii, J. Schmidt, and P. G. Baranov, Phys. Rev. Lett. 88, 045504 (2002).

[9] C. H. Park, S. B. Zhang, and S.-H. Wei, Phys. Rev. B 66, 073202 (2002).

[10] E. A. Meulenkamp, J. Phys. Chem. B 102, 5566 (1998).

[11] Z. Hu, G. Oskam, and P. C. Searson, J. Colloid Interface Sci. 263, 454 (2003).

[12] V. Noack and A. Eychmuller, Chem. Mater. 14, 1411 (2002).

[13] J. A. J. M. Disselhorst, H. J. van der Meer, O. G. Poluektov, and J. Schmidt, J. Magn. Reson., Ser. A 115, 183 (1995).

[14] W. B. Mims, in Electron Paramagnetic Resonance, edited by S. Geschwind (Plenum, New York, 1972).

[15] O. F. Schirmer, J. Phys. Chem. Solids 29, 1407 (1968).

[16] O. F. Schirmer and D. Zwingel, Solid State Commun. 8, 1559 (1970).

[17] H. Zhou, A. Hofstaetter, D. M. Hofmann, and B. K. Meyer, Microelectron. Eng. 66, 59 (2003).

[18] A. V. Rodina, A. L. Efros, M. Rosen, and B. K. Meyer, Mater. Sci. Eng. C 19, 435 (2002).

[19] J. R. Morton and K. F. Preston, J. Magn. Reson. 30, 577 (1978). 